Erzsebet Valeria Hidvegi ${ }^{1}$, Miklos Illyes ${ }^{1}$, Bela Benczur ${ }^{1}$, Renata M. Bocskei ${ }^{1}$, Laszlo Ratgeber ${ }^{2}$, Zsofia Lenkey ${ }^{1}$, Ferenc T. Molnar ${ }^{3}$, Attila Cziraki ${ }^{1}$

${ }^{1}$ Heart Institute, Faculty of Medicine, University of Pecs

2 Faculty of Health Sciences Institute of Public Health and Health Promotion, University of Pecs, Pecs

${ }^{3}$ Department of Hydrodynamic Systems, Budapest University of Technology and Economics, Budapest, Hungary

\title{
Reference Values of Aortic Pulse Wave Velocity in a Large Healthy Population Aged Between 3 and 18 Years
}

Author for correspondence:

Erzsebet Valeria Hidvegi, MD

Address: Heart Institute, Faculty of Medicine, University of Pecs, 13 Ifjusag str., H-7624 Pecs, Hungary,

tel.: +36 56522 301, e-mail: pediatriccor@gmail.com

Accepted: 17.02.2013, submitted for publication: 24.04.2013.

Objective. The measurement of aortic pulse wave velocity $\left(P W V_{a 0}\right)$ is an accepted marker in stratifying individual cardiovascular risk in adults. There is an increasing volume of evidence concerning impaired vascular function in different diseases in paediatric populations, but, unfortunately, only a few studies are available on the measurement of normal $P W V_{a o}$ values in children. The aim of our study was to determine the reference values of $P W V_{a o}$ in a large healthy population using a newly developed technique. Methods. Three thousand, three hundred and seventyfour healthy individuals (1802 boys) aged 3-18 years were examined by an invasively validated, occlusive, oscillometric device. Results. The mean $P W V_{\text {ao }}$ values increased from $5.5 \pm 0.3$ to $6.5 \pm 0.3 \mathrm{~m} / \mathrm{s}(p<00.05)$ in boys and from $5.6 \pm 0.3$ to $6.4 \pm 0.3 \mathrm{~m} / \mathrm{s}$ $(p<0.05)$ in girls. The increase, however, was not constant, and the values exhibited a flat period between the ages of 3 and 8 years in both sexes. The first pronounced increase occurred at the age of 12.1 years in boys and 10.4 years in girls. Moreover, between the ages of 3 and 8 years, the brachial SBP and mean blood pressures increased continuously and gradually, whereas the $P W V_{a o}$ remained unchanged. By contrast, beyond the age of 9 years, blood pressure and aortic stiffness trends basically moved together. Conclusion. Our study provides the largest database to date concerning arterial stiffness in healthy children and adolescents between the ages of 3 and 18 years, and the technology adopted proved easy to use in large paediatric populations, even at a very young age. Abbreviations. CCA, common carotid artery; HR, heart rate; MAP, mean arterial pressure; $P W V_{a o}$, aortic pulse wave velocity; RCA, right carotid artery; SSN, suprasternal notch; TEM, technical errors of measurement.

Key words: aorta, Arteriograph, blood pressure, children and adolescents, pulse wave velocity.

(Pediatricheskaya farmakologiya - Pediatric pharmacology. 2013; 10 (2): 64-71)

\section{INTRODUCTION}

The measurement of arterial stiffness, that is, aortic pulse wave velocity $\left(P W V_{a 0}\right)$, is an accepted marker for detecting organ damage and for stratifyingindividual cardiovascular risk in adults [1]. Moreover, we have an increasing volume of evidence concerning impaired vascular function in different diseases in paediatric populations: early atherosclerosis [2], obesity [3], extreme prematurity [4], familial hypercholesterolemia [5, 6], type I diabetes mellitus [7,8], juvenile hypertension [9], different congenital heart diseases [10, 11], end-stage renal disease [12], HIV infection [13], Kawasaki disease [14], neurofibromatosis [15], vasculitis [16], intrauterine growth restriction patients [17] and primary snoring [18].

These diseases might well influence aortic stiffness, and so the measurement of $\mathrm{PWV}_{\mathrm{ao}}$ may provide useful information, even in a paediatric population. However, to be able to assess the measured $\mathrm{PWV}_{\mathrm{ao}}$ values in different conditions and, further, to assess age-related dynamic changes of $\mathrm{PWV}_{\mathrm{ao}}$ in children and adolescents especially in younger populations of less than 6 years - a precise determination of reference values between the third and 97th percentile is extremely important.

Unfortunately, few studies are available which detail the normal values of $\mathrm{PWV}_{\mathrm{a}}$ in paediatric populations. To date, in fact, a total of 1514 healthy individuals have been studied in six articles [19-24] (Table 1). In the majority of these studies, relatively small populations $(n<140)$ were measured to provide control groups to the diseased populations studied, and it is also worth mentioning that these healthy control groups were remarkably imbalanced in terms of age distribution. Only one study [24] comprised a larger population (1008 individuals, 6-20 years), but even in this study the age distribution of the population was poorly balanced, as a substantial majority (68.1\%) of the individuals studied were from the 15-20 years age group. On the contrary, these studies used ultrasound and applanation tonometry to determine $\mathrm{PWV}_{\mathrm{ao}}$, techniques which are limited in use to the noninvasive clinical evaluation of aortic stiffness in paediatric patients [25].

The aim of our study, therefore, was to determine the reference values of the $\mathrm{PWV}_{\mathrm{ao}}$ in a large healthy

Печатается по: J Hypertens. 2012; 30. 
Table 1. Published normal paediatric aorta pulse wave velocity values

\begin{tabular}{|c|c|c|c|c|c|}
\hline $\begin{array}{l}\text { Age, } \\
\text { years }\end{array}$ & $\begin{array}{c}\text { Number } \\
\text { of participants }\end{array}$ & Method & $\begin{array}{c}\text { Distance } \\
\text { measurement }\end{array}$ & $\mathrm{PWV}_{\mathrm{ao}}, \mathrm{m} / \mathrm{s}$ & Reference \\
\hline 11 & 30 & Pressure transducers & $\mathrm{CCA}$ - a. fem. & $6.5 \pm 1.2$ & Tedesco et al. [19] \\
\hline $12.9 \pm 0.2$ & 110 & Applanation tonometry & $\mathrm{RCA}$ — right a. fem & $4.1-8.2$ & Ahimastos et al. [20] \\
\hline $0.2-20$ & 125 & Doppler ultrasound & CCA - a. fem. & $4.4-7.9$ & Avolio et al. [21] \\
\hline $3-20$ & 108 & Doppler ultrasound & $\begin{array}{c}\text { CCA - a. fem.; } \\
\text { MS - a. fem }\end{array}$ & $6.2-10.2$ & Avolio et al. [22] \\
\hline $6-23$ & 133 & Applanation tonometry & CCA-SSN+SSN - a. fem & $5.02 \pm 0.89$ & Kis et al. [23] \\
\hline $6-20$ & $\begin{array}{l}495 \text { male; } \\
513 \text { female }\end{array}$ & Applanation tonometry & CCA-SSN-SSN - a. fem & $\begin{array}{l}\text { Male 4.34-5.71; } \\
\text { female 4.34-5.50 }\end{array}$ & Reusz et al. [24] \\
\hline
\end{tabular}

a. fem — femoral artery; CCA — common carotid artery; MS — manubrium sterni; RCA — right carotid artery; SSN — suprasternal notch.

Table 2. Intraobserver and interobserver errors

\begin{tabular}{|c|c|c|c|c|c|c|c|c|c|c|c|c|c|c|c|}
\hline \multirow{2}{*}{ Physician(s) } & \multicolumn{5}{|c|}{ Intraobserver } & \multicolumn{10}{|c|}{ Interobserver } \\
\hline & 1 & 2 & 3 & 4 & 5 & $1-2$ & $1-3$ & $1-4$ & $1-5$ & $2-3$ & $2-4$ & $2-5$ & $3-4$ & $3-5$ & 4-5 \\
\hline TEM (cm) & 0.23 & 0.31 & 0.26 & 0.28 & 0.20 & 0.27 & 0.27 & 0.34 & 0.30 & 0.30 & 0.32 & 0.32 & 0.32 & 0.27 & 0.30 \\
\hline Relative TEM (\%) & 0.5 & 0.7 & 0.5 & 0.6 & 0.4 & 0.6 & 0.6 & 0.7 & 0.6 & 0.6 & 0.7 & 0.7 & 0.7 & 0.6 & 0.6 \\
\hline
\end{tabular}

TEM - technicalerrorofmeasurement.

population which showed a fair balance in terms of age. We intended to apply a newly developed, invasively validated and easytouse oscillometric technique which allowed us to expand measurement even into a very young ( $<6$ years) population and within a daily clinical routine.

\section{METHODS}

\section{Participants}

Three thousand, three hundred and seventyfour apparently healthy white individuals without any medication and/or blood pressure-affecting drugs from Hungary (1802 boys and 1572 girls) aged 3-18 years, having BMI and SBP and DBP within the third and 97th percentiles, as established by the relevant guidelines $[26,27]$, were recruited from nursery, elementary and secondary schools. Written consent for the measurements to be taken was given by the parents. The protocol of this clinical study was reviewed and approved by the local Institutional Ethics Committee of the University of Pecs, Pecs, Hungary.

\section{Methods}

A noninvasive, occlusive, oscillometric device (Arteriograph; TensioMed Ltd, Budapest, Hungary) was used for the $\mathrm{PWV}_{\text {ao }}$ measurements, for which the operating procedure did not, in practice, differ from a standard digital blood pressure measurement. The method and its validations have been detailed previously $[28,29]$. Briefly, the method is based on the physiological fact that the early $\left(\mathrm{P}_{1}\right)$ systolic pulse pressure wave of the aorta, generated by the left ventricle ejection, travels along the aorta and is reflected $\left(\mathrm{P}_{2}\right)$ from the area of the aortic bifurcation. Occluding the brachial artery by pressurizing the cuff $35-40 \mathrm{mmHg}$ above the actual SBP creates easily distinguished, pronounced pressure peaks in the cuff. Separated in this way, early and late systolic waves can be recorded. The time lapses between the peaks of
$\mathrm{P}_{1}$ and $\mathrm{P}_{2}$ are equal to the travel time of the aortic pressure wave from the aortic root to the bifurcation and back. By halving this time and measuring the sternal notch - pubic bone distance (which is rather close to the true aortic length [30]), the $\mathrm{PWV}_{\mathrm{ao}}$ can be calculated $\left(\mathrm{PWV}_{\mathrm{ao}}=\right.$ jjugulumsymphysis distance/transit time). The $\mathrm{PWV}_{\mathrm{ao}}$ measurements were taken with the participant in a calm, supine position after several minutes of rest.

The interobserver and intraobserver errors of the distance (sternal notch-pubic bone) measurements were expressed as absolute and relative technical errors of measurement (TEM) [31] and proved to be well within acceptable limits (Table 2 ).

\section{Data analysis and statistics}

Data are reported as mean and SD for continuous data. For data comparison, a Student's t-test was used after checking that the assumption of normality was met. A significance level of 0.05 was used for statistical tests. Statistical analysis was performed with the SPSS 15.0 statistical package (SPSS Inc., Chicago, Illinois, USA).

Reference percentile curves were obtained with the use of the LMS method [32], specifically with the LMS Chartmaker software [33]. The method summarizes the distribution of a variable by its skewness (expressed as Box-Cox power, $\mathrm{L}$ ), its median (M) and its coefficient of variation (S). The agedependent changes in these parameters are smoothed by fitting cubic splines with appropriately selected smoothing parameters, using penalized likelihood.

\section{RESULTS}

The characteristics of the subjects investigated are summarized in Tables 3 and 4. Both the boys and the girls are fairly well balanced in terms of age, whereas $15 \%$ of the population was recruited from very young age groups (3-6 years). 
Table 3. Characteristics of boys

\begin{tabular}{|c|c|c|c|c|c|c|c|c|c|c|}
\hline $\begin{array}{c}\text { Age } \\
\text { (years) }\end{array}$ & $n$ & $\begin{array}{l}\text { Height } \\
\text { (cm) }\end{array}$ & $\begin{array}{l}\text { Weight } \\
\text { (kg) }\end{array}$ & $\begin{array}{l}\text { BSA } \\
\left(\mathrm{m}^{2}\right)\end{array}$ & BMI (\%) & $\begin{array}{l}\text { SBP }_{\text {brach }} \\
\text { (mmHg) }\end{array}$ & $\begin{array}{l}\text { DBP }_{\text {brach }} \\
\text { (mmHg) }\end{array}$ & $\begin{array}{c}\text { MAP } \\
(\mathrm{mmHg})\end{array}$ & $\begin{array}{c}\text { HR } \\
\text { (beats/min) }\end{array}$ & $\begin{array}{c}\text { JUG-SY } \\
\text { (cm) }\end{array}$ \\
\hline 03 & 044 & $104.4 \pm 6.7$ & $16.2 \pm 02.5$ & $0.7 \pm 0.1$ & $14.8 \pm 1.9$ & $103.0 \pm 5.5$ & $61.8 \pm 7.3$ & $76.5 \pm 7.5$ & $98.7 \pm 11.1$ & $32.0 \pm 2.5$ \\
\hline 04 & 053 & $108.6 \pm 7.6$ & $17.5 \pm 02.7$ & $0.7 \pm 0.1$ & $14.8 \pm 1.6$ & $104.1 \pm 7.7$ & $61.5 \pm 7.5$ & $76.9 \pm 8.5$ & $92.5 \pm 13.0$ & $33.1 \pm 2.9$ \\
\hline 05 & 080 & $115.5 \pm 6.8$ & $19.7 \pm 02.9$ & $0.8 \pm 0.1$ & $14.8 \pm 1.7$ & $106.4 \pm 7.2$ & $62.7 \pm 6.3$ & $78.3 \pm 7.8$ & $91.5 \pm 13.4$ & $35.0 \pm 2.2$ \\
\hline 06 & 120 & $123.2 \pm 6.2$ & $22.4 \pm 03.5$ & $0.9 \pm 0.1$ & $14.7 \pm 1.6$ & $104.5 \pm 6.8$ & $62.0 \pm 6.3$ & $76.3 \pm 6.4$ & $86.6 \pm 11.5$ & $37.2 \pm 2.7$ \\
\hline 07 & 085 & $127.8 \pm 6.8$ & $25.2 \pm 04.2$ & $0.9 \pm 0.1$ & $15.4 \pm 1.8$ & $107.8 \pm 7.2$ & $63.8 \pm 6.3$ & $78.8 \pm 6.7$ & $83.7 \pm 13.1$ & $39.0 \pm 2.7$ \\
\hline 08 & 074 & $132.9 \pm 8.0$ & $27.9 \pm 04.7$ & $1.0 \pm 0.1$ & $15.8 \pm 1.8$ & $106.9 \pm 7.1$ & $62.8 \pm 5.3$ & $77.4 \pm 5.4$ & $79.9 \pm 13.2$ & $40.0 \pm 2.9$ \\
\hline 09 & 092 & $138.5 \pm 7.4$ & $31.2 \pm 05.9$ & $1.1 \pm 0.1$ & $16.1 \pm 1.9$ & $109.3 \pm 6.9$ & $64.8 \pm 6.8$ & $79.2 \pm 6.1$ & $79.7 \pm 13.5$ & $42.0 \pm 3.3$ \\
\hline 10 & 081 & $144.5 \pm 6.6$ & $36.1 \pm 06.5$ & $1.2 \pm 0.1$ & $17.2 \pm 2.2$ & $111.2 \pm 7.3$ & $64.7 \pm 5.5$ & $80.5 \pm 5.7$ & $78.7 \pm 12.3$ & $44.0 \pm 3.4$ \\
\hline 11 & 080 & $148.4 \pm 8.4$ & $38.9 \pm 07.8$ & $1.3 \pm 0.2$ & $17.5 \pm 2.4$ & $111.5 \pm 7.5$ & $65.2 \pm 5.7$ & $80.7 \pm 5.9$ & $73.7 \pm 11.5$ & $44.3 \pm 3.3$ \\
\hline 12 & 101 & $155.3 \pm 9.6$ & $44.3 \pm 09.3$ & $1.4 \pm 0.2$ & $18.2 \pm 2.5$ & $115.0 \pm 7.5$ & $65.3 \pm 6.3$ & $82.1 \pm 6.1$ & $77.5 \pm 13.8$ & $47.2 \pm 4.2$ \\
\hline 13 & 169 & $163.1 \pm 9.5$ & $50.8 \pm 09.3$ & $1.5 \pm 0.2$ & $19.0 \pm 2.3$ & $117.2 \pm 7.8$ & $64.8 \pm 6.3$ & $82.3 \pm 6.1$ & $76.3 \pm 12.6$ & $49.2 \pm 3.7$ \\
\hline 14 & 187 & $169.0 \pm 9.8$ & $55.5 \pm 09.8$ & $1.6 \pm 0.2$ & $19.3 \pm 2.4$ & $119.5 \pm 7.4$ & $65.7 \pm 5.4$ & $83.7 \pm 5.2$ & $76.2 \pm 13.2$ & $51.0 \pm 3.4$ \\
\hline 15 & 171 & $174.1 \pm 7.6$ & $59.9 \pm 09.2$ & $1.7 \pm 0.2$ & $19.7 \pm 2.4$ & $121.1 \pm 8.0$ & $67.5 \pm 6.2$ & $85.4 \pm 6.0$ & $71.3 \pm 12.8$ & $51.8 \pm 3.4$ \\
\hline 16 & 162 & $175.9 \pm 7.1$ & $64.3 \pm 11.2$ & $1.8 \pm 0.2$ & $20.7 \pm 2.8$ & $124.5 \pm 8.5$ & $68.6 \pm 6.2$ & $87.3 \pm 6.2$ & $72.5 \pm 14.3$ & $53.1 \pm 3.3$ \\
\hline 17 & 197 & $178.0 \pm 7.2$ & $67.5 \pm 10.2$ & $1.8 \pm 0.2$ & $21.3 \pm 2.7$ & $126.3 \pm 8.6$ & $68.8 \pm 6.5$ & $88.0 \pm 6.2$ & $69.9 \pm 11.9$ & $54.5 \pm 3.4$ \\
\hline 18 & 106 & $179.6 \pm 6.9$ & $69.6 \pm 08.7$ & $1.9 \pm 0.1$ & $21.6 \pm 2.4$ & $128.2 \pm 8.3$ & $67.4 \pm 7.3$ & $87.7 \pm 6.4$ & $70.3 \pm 13.2$ & $55.3 \pm 3.5$ \\
\hline
\end{tabular}

Data are shown as mean \pm SD. DBP $_{\text {brach }}$ - brachial DBP; HR — heart rate; JUG-SY — distance between sternal notch (jugulum) and upper part of the pubic bone (symphysis); MAP — mean arterial pressure; $\mathrm{SBP}_{\text {brach }}$ - brachial SBP.

Table 4. Characteristics of girls

\begin{tabular}{|c|c|c|c|c|c|c|c|c|c|c|}
\hline $\begin{array}{c}\text { Age } \\
(\mathbf{y e a r s})\end{array}$ & $\boldsymbol{n}$ & Height $\mathbf{( \mathbf { c m } )}$ & $\begin{array}{c}\text { Weight } \\
\mathbf{( k g )}\end{array}$ & $\mathbf{B S A}\left(\mathbf{m}^{\mathbf{2}} \mathbf{)}\right.$ & $\mathbf{B M I}(\mathbf{\%})$ & $\begin{array}{c}\mathbf{S B P}_{\text {brach }} \\
\mathbf{( m m H g})\end{array}$ & $\begin{array}{c}\mathbf{D B P}_{\text {brach }} \\
(\mathbf{m m H g})\end{array}$ & $\begin{array}{c}\text { MAP } \\
(\mathbf{m m H g})\end{array}$ & $\begin{array}{c}\text { HR } \\
\text { (beats/min) }\end{array}$ & $\begin{array}{c}\text { JUG-SY } \\
(\mathbf{c m})\end{array}$ \\
\hline 03 & 035 & $102.2 \pm 06.3$ & $15.5 \pm 2.4$ & $0.7 \pm 0.1$ & $14.8 \pm 1.2$ & $102.5 \pm 5.1$ & $61.1 \pm 9.4$ & $75.4 \pm 8.1$ & $101.1 \pm 14.4$ & $30.9 \pm 1.9$ \\
\hline 04 & 043 & $107.1 \pm 06.4$ & $17.1 \pm 2.9$ & $0.7 \pm 0.1$ & $14.8 \pm 1.8$ & $103.3 \pm 5.2$ & $60.0 \pm 5.2$ & $75.2 \pm 4.9$ & $092.4 \pm 11.6$ & $31.8 \pm 2.8$ \\
\hline 05 & 044 & $114.5 \pm 07.4$ & $18.8 \pm 3.3$ & $0.8 \pm 0.1$ & $14.3 \pm 1.7$ & $103.7 \pm 6.3$ & $61.7 \pm 7.2$ & $76.1 \pm 6.9$ & $093.7 \pm 11.7$ & $33.4 \pm 2.1$ \\
\hline 06 & 068 & $123.0 \pm 06.6$ & $22.2 \pm 4.0$ & $0.9 \pm 0.1$ & $14.6 \pm 1.7$ & $105.0 \pm 6.9$ & $62.1 \pm 5.8$ & $76.8 \pm 6.7$ & $087.3 \pm 13.3$ & $35.9 \pm 2.4$ \\
\hline 07 & 072 & $128.6 \pm 10.3$ & $25.2 \pm 7.1$ & $0.9 \pm 0.2$ & $15.0 \pm 2.3$ & $106.4 \pm 7.3$ & $63.2 \pm 5.6$ & $77.7 \pm 6.0$ & $086.5 \pm 12.5$ & $38.2 \pm 3.3$ \\
\hline 08 & 039 & $133.9 \pm 07.1$ & $28.1 \pm 5.7$ & $1.0 \pm 0.1$ & $15.6 \pm 2.4$ & $107.6 \pm 7.3$ & $63.6 \pm 4.6$ & $78.3 \pm 5.0$ & $083.2 \pm 11.1$ & $39.2 \pm 2.3$ \\
\hline 09 & 064 & $138.6 \pm 08.1$ & $31.9 \pm 6.5$ & $1.1 \pm 0.1$ & $16.5 \pm 2.3$ & $109.6 \pm 6.4$ & $64.8 \pm 5.8$ & $79.3 \pm 5.4$ & $084.9 \pm 11.7$ & $40.8 \pm 3.1$ \\
\hline 10 & 063 & $144.0 \pm 07.1$ & $34.4 \pm 6.6$ & $1.2 \pm 0.1$ & $16.5 \pm 2.3$ & $111.1 \pm 7.3$ & $64.8 \pm 5.5$ & $80.2 \pm 5.5$ & $082.5 \pm 09.4$ & $42.4 \pm 3.2$ \\
\hline 11 & 049 & $150.8 \pm 09.8$ & $39.5 \pm 8.4$ & $1.3 \pm 0.2$ & $17.2 \pm 2.5$ & $112.1 \pm 6.8$ & $65.4 \pm 5.0$ & $81.0 \pm 5.0$ & $080.1 \pm 09.4$ & $44.2 \pm 3.7$ \\
\hline 12 & 085 & $156.8 \pm 07.8$ & $44.6 \pm 8.8$ & $1.4 \pm 0.2$ & $18.0 \pm 2.5$ & $113.4 \pm 7.5$ & $65.6 \pm 5.4$ & $81.5 \pm 5.6$ & $079.5 \pm 12.8$ & $46.2 \pm 3.5$ \\
\hline 13 & 195 & $160.8 \pm 07.7$ & $49.6 \pm 7.9$ & $1.5 \pm 0.1$ & $19.1 \pm 2.6$ & $115.1 \pm 7.6$ & $65.4 \pm 6.2$ & $82.0 \pm 5.9$ & $078.6 \pm 13.8$ & $47.8 \pm 3.0$ \\
\hline 14 & 189 & $163.7 \pm 06.5$ & $52.3 \pm 8.3$ & $1.5 \pm 0.1$ & $19.5 \pm 2.6$ & $117.6 \pm 8.2$ & $67.0 \pm 6.2$ & $83.8 \pm 6.3$ & $077.7 \pm 14.2$ & $48.7 \pm 2.8$ \\
\hline 15 & 181 & $164.8 \pm 08.0$ & $54.8 \pm 9.3$ & $1.6 \pm 0.2$ & $20.1 \pm 2.7$ & $117.2 \pm 7.6$ & $68.5 \pm 5.8$ & $84.7 \pm 5.9$ & $077.1 \pm 12.3$ & $48.6 \pm 3.4$ \\
\hline 16 & 174 & $164.9 \pm 06.7$ & $55.8 \pm 8.5$ & $1.6 \pm 0.1$ & $20.5 \pm 2.7$ & $117.8 \pm 7.4$ & $69.2 \pm 5.4$ & $85.4 \pm 5.5$ & $074.4 \pm 11.7$ & $49.1 \pm 3.2$ \\
\hline 17 & 175 & $165.9 \pm 06.3$ & $56.2 \pm 9.3$ & $1.6 \pm 0.1$ & $20.4 \pm 2.9$ & $117.7 \pm 8.0$ & $68.0 \pm 6.0$ & $84.5 \pm 6.1$ & $074.7 \pm 10.2$ & $49.8 \pm 3.1$ \\
\hline 18 & 096 & $166.3 \pm 06.6$ & $57.5 \pm 8.4$ & $1.6 \pm 0.1$ & $20.8 \pm 2.8$ & $118.8 \pm 8.6$ & $68.2 \pm 6.0$ & $85.1 \pm 6.3$ & $077.0 \pm 11.0$ & $51.2 \pm 2.8$ \\
\hline
\end{tabular}

Data are shown as mean \pm SD. DBP brach — brachial DBP; HR - heart rate; JUG-SY — distance between sternal notch (jugulum) and upper part of the pubic bone (symphysis); MAP — mean arterial pressure; $\mathrm{SBP}_{\text {brach }}$ - brachial SBP.

All of the parameters measured showed a marked increase with age, except for the heart rate - which decreased, as physiologically expected.

Table 5 summarizes the $\mathrm{PWV}_{\mathrm{ao}}$ changes. The increase of mean $\mathrm{PWV}_{\mathrm{ao}}$ values turned out to be roughly $1 \mathrm{~m} / \mathrm{s}$ in both sexes from the age of 3 to 18 years, as this parameter increased from $5.5 \pm 0.3$ to $6.5 \pm 0.3 \mathrm{~m} / \mathrm{s}(p<0.05)$ in boys and from $5.6 \pm 0.3$ to $6.4 \pm 0.3 \mathrm{~m} / \mathrm{s}(p<0.05)$ in girls. When comparing the mean $\mathrm{PWV}_{\mathrm{ao}}$ values in each year between boys and girls, noticeable differences were found in the 
Table 5. Normal aortic pulse wave velocity mean values in boys and girls

\begin{tabular}{|c|c|c|c|c|c|}
\hline \multirow{2}{*}{ Age (years) } & \multicolumn{2}{|c|}{ Boys } & \multicolumn{2}{|c|}{ Girls } & \multirow{2}{*}{$\begin{array}{c}\text { Student's } \\
\text { t-test }\end{array}$} \\
\hline & $n$ & $\mathrm{PWV}_{\mathrm{ao}}(\mathrm{m} / \mathrm{s}) \pm \mathrm{SD}$ & $n$ & $\mathrm{PWV}_{\mathrm{ao}}(\mathrm{m} / \mathrm{s}) \pm \mathrm{SD}$ & \\
\hline 03 & 044 & $5.5 \pm 0.6$ & 035 & $5.6 \pm 0.6$ & NS \\
\hline 04 & 053 & $5.5 \pm 0.6$ & 043 & $5.3 \pm 0.6$ & NS \\
\hline 05 & 080 & $5.5 \pm 0.6$ & 044 & $5.4 \pm 0.8$ & NS \\
\hline 06 & 120 & $5.4 \pm 0.7$ & 068 & $5.3 \pm 0.7$ & NS \\
\hline 07 & 085 & $5.5 \pm 0.5$ & 072 & $5.5 \pm 0.6$ & NS \\
\hline 08 & 074 & $5.4 \pm 0.6$ & 039 & $5.4 \pm 0.6$ & NS \\
\hline 09 & 092 & $5.6 \pm 0.6$ & 064 & $5.5 \pm 0.6$ & NS \\
\hline 10 & 081 & $5.5 \pm 0.7$ & 063 & $5.7 \pm 0.7$ & NS \\
\hline 11 & 080 & $5.6 \pm 0.7$ & 049 & $5.7 \pm 0.7$ & NS \\
\hline 12 & 101 & $5.8 \pm 0.7$ & 085 & $5.8 \pm 0.5$ & NS \\
\hline 13 & 169 & $5.8 \pm 0.6$ & 195 & $5.9 \pm 0.6$ & NS \\
\hline 14 & 187 & $6.0 \pm 0.7$ & 189 & $5.9 \pm 0.6$ & $p<0.05$ \\
\hline 15 & 171 & $6.0 \pm 0.6$ & 181 & $6.0 \pm 0.6$ & NS \\
\hline 16 & 162 & $6.2 \pm 0.6$ & 174 & $6.0 \pm 0.5$ & $p<0.004$ \\
\hline 17 & 197 & $6.3 \pm 0.6$ & 175 & $6.2 \pm 0.5$ & NS \\
\hline 18 & 106 & $6.5 \pm 0.5$ & 096 & $6.4 \pm 0.5$ & NS \\
\hline
\end{tabular}

$\mathrm{PWV}_{\mathrm{ao}}$ - aortic pulse wave velocity.

FIGURE 1. Smoothed percentile curves from third to 97 th of aortic pulse wave velocity $\left(P W V_{a 0}\right)$ related with age for boys $(A)$ and girls $(B)$

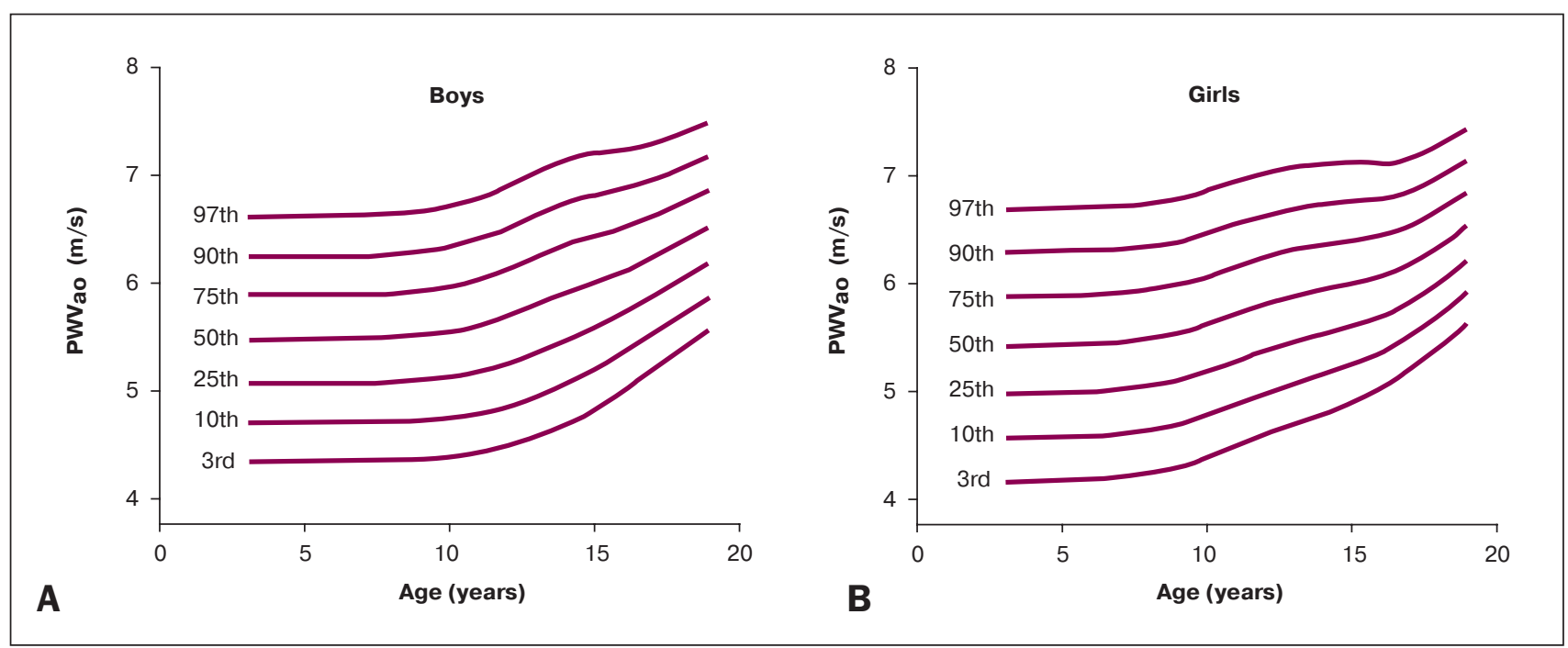

14-year and 16-year age groups. However, these differences are merely 0.1 and $0.2 \mathrm{~m} / \mathrm{s}$, respectively, and so cannot be considered as clinically significant.

Smoothed percentile curves of $\mathrm{PWV}_{\mathrm{a}}$ from the third to the 97th are shown for boys and girls in Fig. 1, and the corresponding values of $\mathrm{PWV}_{\mathrm{ao}}$ can be seen in Tables 6 and 7 .

Analysing the curves of the median values of $\mathrm{PWV}_{\mathrm{ao}}$ in boys and girls, it became clear that the increase was not constant with age, but had more complex dynamics (Fig. 1). In both sexes, the PWV ${ }_{\text {ao }}$ values exhibit a flat period between 3 and 8 years, with a steeper increase thereafter. The first derivative of the smoothed median curve of $\mathrm{PWV}_{\text {ao }}$ showed that the first pronounced increase occurred at the age of 12.1 years in boys and 10.4 years in girls (Fig. 2).

To study causal relationships between $\mathrm{PWV}_{\mathrm{ao}}$ and other variables, graphical analyses were used, as multivariate linear regression cannot be applied due to the high multicollinearity among age, anthropometric and hemodynamic parameters. In Fig. 3, we demonstrate the relationship between $\mathrm{PWV}_{\mathrm{ao}}$, mean arterial pressure (MAP) and brachial SBP $\left(\mathrm{SBP}_{\text {brach }}\right)$, featuring the median of the standardizedvalues of the parameters mentioned in boys and girls. Interestingly, at ages between 3 and 8 years a marked difference was observed in the trend of the 


\begin{tabular}{|c|c|c|c|c|c|c|c|c|c|c|c|c|c|}
\hline$\stackrel{\infty}{\sim}$ & 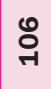 & $\dashv$ & $\begin{array}{c}\tilde{N} \\
\infty \\
\tilde{\omega} \\
\omega\end{array}$ & $\mid \begin{array}{l}m \\
\infty \\
0 \\
0 \\
0\end{array}$ & 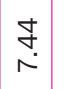 & 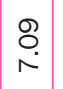 & $\begin{array}{l}m \\
\stackrel{0}{0}\end{array}$ & $\begin{array}{l}\infty \\
m \\
0 \\
\dot{0}\end{array}$ & $\begin{array}{l}m \\
0 \\
\dot{0}\end{array}$ & \begin{tabular}{|c|}
$\infty$ \\
0 \\
0 \\
\llcorner
\end{tabular} & $\begin{array}{c}\tilde{N} \\
m \\
i\end{array}$ & $\begin{array}{l}0 \\
0 \\
\stackrel{T}{r}\end{array}$ & $\begin{array}{l}0 \\
8 \\
0\end{array}$ \\
\hline ન & ลิ & $\dashv$ & $\mid \begin{array}{l}0 \\
⿱ 亠 乂 \\
0 \\
0\end{array}$ & $\mid \begin{array}{l}0 \\
\infty \\
0 \\
0\end{array}$ & $\stackrel{\stackrel{\rho}{m}}{\stackrel{m}{r}}$ & $\begin{array}{l}\infty \\
\stackrel{\infty}{ } \\
\dot{0}\end{array}$ & $\begin{array}{l}-1 \\
\dot{0} \\
\dot{0}\end{array}$ & 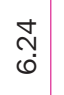 & $\begin{array}{l}\hat{\infty} \\
\infty \\
\omega^{\prime}\end{array}$ & $\begin{array}{l}0 \\
\stackrel{\circ}{0} \\
\llcorner\end{array}$ & $\begin{array}{l}m \\
\stackrel{n}{n} \\
\text { | }\end{array}$ & $\begin{array}{l}-1 \\
\infty \\
\stackrel{-}{\sigma} \\
-1\end{array}$ & $\begin{array}{l}\stackrel{0}{1} \\
\hat{0}\end{array}$ \\
\hline$\stackrel{\oplus}{-1}$ & స్త & $\dashv$ & $\begin{array}{l}0 \\
\stackrel{-}{-} \\
\stackrel{-}{0} \\
0\end{array}$ & $\begin{array}{l}\hat{\sigma} \\
\circ \\
0 \\
0\end{array}$ & 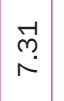 & नু & $\begin{array}{l}-1 \\
0 \\
0\end{array}$ & $\begin{array}{l}\text { フै } \\
6\end{array}$ & $\begin{array}{l}N \\
N \\
\llcorner \\
0\end{array}$ & $\begin{array}{c}\tilde{N} \\
\tilde{\omega} \\
\tilde{D}\end{array}$ & $\begin{array}{l}\mathscr{N} \\
\stackrel{+}{+}\end{array}$ & 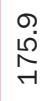 & $\begin{array}{l}m \\
\dot{P}\end{array}$ \\
\hline$\stackrel{19}{\rightarrow}$ & $\underset{7}{\stackrel{-}{7}}$ & $\dashv$ & $\begin{array}{l}0 \\
-1 \\
0 \\
\dot{0}\end{array}$ & $\begin{array}{c}1 \\
0 \\
\stackrel{1}{\circ} \\
0\end{array}$ & 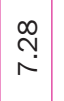 & $\left|\begin{array}{l}1 \\
\infty \\
\dot{0}\end{array}\right|$ & 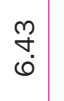 & $\begin{array}{l}-1 \\
\dot{0}\end{array}$ & 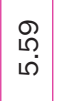 & $\begin{array}{c}\text { ने } \\
\text { ம் }\end{array}$ & $\begin{array}{l}\stackrel{+}{\sim} \\
\dot{+}\end{array}$ & $\underset{+}{\stackrel{-}{+}}$ & $\begin{array}{l}\mathscr{8} \\
\dot{\circ}\end{array}$ \\
\hline 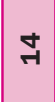 & 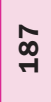 & $\dashv$ & $\begin{array}{l}\stackrel{-1}{-1} \\
\stackrel{\sigma}{\circ} \\
\dot{0}\end{array}$ & $\begin{array}{c}0 \\
-1 \\
\\
0 \\
0\end{array}$ & $\underset{N}{\stackrel{N}{N}}$ & $\begin{array}{l}\infty \\
\stackrel{0}{0} \\
\dot{0}\end{array}$ & $\begin{array}{l}\stackrel{\llcorner}{m} \\
\ddot{0}\end{array}$ & $\begin{array}{l}-1 \\
\text { م่ }\end{array}$ & $\begin{array}{l}\infty \\
+ \\
\vdots \\
1\end{array} \mid$ & 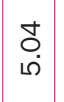 & $\begin{array}{l}\overrightarrow{0} \\
\dot{+}\end{array}$ & $\begin{array}{l}\circ \\
\stackrel{\circ}{0} \\
\stackrel{6}{-1}\end{array}$ & 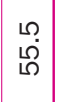 \\
\hline$\stackrel{m}{\rightarrow}$ & $\underset{్}{\infty}$ & $\dashv$ & $\begin{array}{l}0 \\
\stackrel{9}{1} \\
\dot{1}\end{array}$ & $\begin{array}{c}m \\
\rightarrow \\
\\
0 \\
0\end{array}$ & $\stackrel{\overbrace{}}{\stackrel{ }{\sim}}$ & $\begin{array}{l}\hat{\varphi} \\
\dot{0}\end{array}$ & 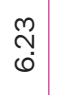 & $\begin{array}{l}0 \\
\infty \\
\omega^{\circ}\end{array}$ & $\begin{array}{c}0 \\
\tilde{m} \\
\omega\end{array}$ & $\begin{array}{l}\tilde{\sigma} \\
\dot{\tau} \\
\end{array}$ & $\begin{array}{l}\stackrel{g}{+} \\
\dot{+}\end{array}$ & $\begin{array}{r}-1 \\
\ddot{\theta} \\
\stackrel{-}{0}\end{array}$ & $\begin{array}{l}\infty \\
\dot{0} \\
10\end{array}$ \\
\hline ส & -1 & $\dashv$ & $\begin{array}{c}m \\
0 \\
0 \\
10 \\
10\end{array}$ & $\begin{array}{c}m \\
7 \\
7 \\
0\end{array}$ & $\begin{array}{l}\hat{\Omega} \\
\text { ஸे }\end{array}$ & $\begin{array}{l}\dot{0} \\
\stackrel{1}{0} \\
\dot{0}\end{array}$ & 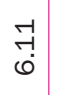 & $\begin{array}{l}\infty \\
0 \\
\llcorner \\
ம^{\circ}\end{array}$ & $\stackrel{\stackrel{\sim}{N}}{\sim}$ & $\begin{array}{c}N \\
\infty \\
\dot{\sigma}\end{array} \mid$ & $\begin{array}{l}\stackrel{P}{+} \\
\dot{T}\end{array}$ & $\begin{array}{l}m \\
\stackrel{m}{\rho} \\
\stackrel{\sim}{\sim}\end{array}$ & $\begin{array}{l}m \\
\dot{f} \\
\end{array}$ \\
\hline$\exists$ & $\vec{\infty}$ & 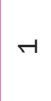 & 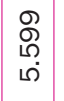 & 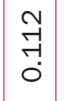 & $\begin{array}{l}0 \\
\infty \\
0\end{array}$ & $\begin{array}{l}7 \\
\dot{0} \\
0\end{array}$ & $\begin{array}{l}\mathrm{N} \\
0 \\
0\end{array}$ & $\begin{array}{c}8 \\
\Theta \\
1\end{array}$ & $\begin{array}{c}\infty \\
\stackrel{-}{-1} \\
\stackrel{0}{0}\end{array} \mid$ & $\begin{array}{l}0 \\
\sim \\
\dot{\sigma}\end{array}$ & $\begin{array}{l}\text { mे } \\
\dot{\sigma}\end{array}$ & $\begin{array}{l}\overrightarrow{+} \\
\infty \\
\stackrel{+}{+}\end{array}$ & $\begin{array}{l}\infty \\
\infty \\
\infty\end{array}$ \\
\hline 우 & $\infty$ & H & $\begin{array}{l}\stackrel{9}{+} \\
\llcorner \\
\llcorner \\
\llcorner\end{array}$ & $\begin{array}{l}-1 \\
7 \\
-1 \\
0\end{array}$ & $\begin{array}{l}\stackrel{9}{T} \\
\dot{0}\end{array}$ & $\begin{array}{c}\hat{m} \\
\dot{0}\end{array}$ & $\begin{array}{l}0 \\
\stackrel{\circ}{\circ} \\
\text { ம) }\end{array}$ & 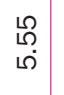 & 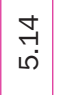 & $\begin{array}{l}\mathfrak{N} \\
\dot{\sigma}\end{array}$ & $\begin{array}{l}\vec{m} \\
\dot{+}\end{array}$ & 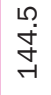 & $\begin{array}{l}-1 \\
\dot{e}\end{array}$ \\
\hline a & สู & -1 & $\begin{array}{l}\vec{J} \\
\stackrel{\llcorner}{0} \\
\llcorner 0\end{array}$ & 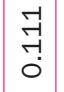 & $\begin{array}{l}\stackrel{t}{*} \\
\dot{0}\end{array}$ & $\begin{array}{l}m \\
m \\
\dot{\theta}\end{array}$ & $\begin{array}{l}\tilde{N} \\
\text { ஸे }\end{array}$ & $\begin{array}{l}\stackrel{-1}{\llcorner} \\
\llcorner \\
\llcorner\end{array}$ & $\begin{array}{l}\stackrel{-}{-} \\
\stackrel{-}{0}\end{array}$ & $\begin{array}{l}\stackrel{P}{r} \\
\dot{r}\end{array} \mid$ & $\underset{⿱}{\stackrel{\sim}{+}}$ & $\begin{array}{l}\stackrel{1}{0} \\
\infty \\
\stackrel{m}{\rightarrow}\end{array}$ & Nָ \\
\hline$\infty$ & t & $\dashv$ & $\begin{array}{l}\text { Oे } \\
\stackrel{+}{ } \\
\text { ம் }\end{array}$ & $\begin{array}{l}-1 \\
7 \\
7 \\
0\end{array}$ & $\begin{array}{l}\overrightarrow{+} \\
\dot{0}\end{array}$ & $\begin{array}{l}0 \\
m \\
\dot{0}\end{array}$ & $\begin{array}{l}\circ \\
\text { வे }\end{array}$ & $\begin{array}{l}\stackrel{g}{+} \\
\text { மீ) }\end{array}$ & $\begin{array}{l}8 \\
0 \\
10\end{array}$ & $\begin{array}{l}\infty \\
0 \\
\dot{\tau}\end{array}$ & $\begin{array}{l}\stackrel{\infty}{\sim} \\
\stackrel{+}{*}\end{array}$ & $\begin{array}{l}\stackrel{\wp}{\sim} \\
\underset{\sim}{\sim}\end{array}$ & $\stackrel{?}{\stackrel{?}{N}}$ \\
\hline$\sim$ & $\stackrel{\llcorner}{\infty}$ & -1 & $\begin{array}{c}m \\
\infty \\
0 \\
+ \\
\infty\end{array}$ & $\begin{array}{l}0 \\
7 \\
7 \\
0\end{array}$ & $\begin{array}{l}\mathscr{8} \\
\dot{0}\end{array}$ & $\stackrel{\mathscr{N}}{\mathbf{0}}$ & $\begin{array}{l}\infty \\
\infty \\
\llcorner\end{array}$ & $\begin{array}{l}\stackrel{\infty}{+} \\
\stackrel{0}{\circ}\end{array}$ & $\begin{array}{c}\infty \\
0 \\
1 \\
\omega^{2}\end{array}$ & $\mid \begin{array}{l}\infty \\
0 \\
\dot{\tau}\end{array}$ & $\stackrel{N}{\sim}$ & 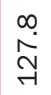 & $\begin{array}{c}\stackrel{N}{\stackrel{N}{N}} \\
\text {. }\end{array}$ \\
\hline 0 & กิ & $\dashv$ & $\begin{array}{c}N \\
\infty \\
+ \\
+ \\
\omega\end{array}$ & $\begin{array}{c}0 \\
-1 \\
7 \\
0\end{array}$ & $\begin{array}{l}8 \\
0 \\
0\end{array}$ & 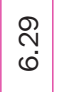 & $\begin{array}{l}\mathscr{8} \\
\infty \\
\dot{1}\end{array}$ & $\begin{array}{l}\stackrel{\infty}{+} \\
\stackrel{0}{0}\end{array}$ & $\begin{array}{l}\infty \\
0 \\
10\end{array}$ & $\begin{array}{l}\hat{\varphi} \\
\dot{\tau}\end{array}$ & $\begin{array}{l}\hat{N} \\
\dot{f}\end{array}$ & Nָ & $\stackrel{+}{\grave{N}}$ \\
\hline เ) & ০ & $\dashv$ & $\begin{array}{c}9 \\
\stackrel{T}{+} \\
\dot{+}\end{array}$ & 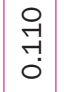 & $\begin{array}{l}\mathscr{8} \\
0 \\
0\end{array}$ & $\begin{array}{l}\stackrel{\mathscr{N}}{0} \\
\dot{0}\end{array}$ & $\begin{array}{l}\infty \\
\infty \\
\omega^{\circ}\end{array}$ & $\begin{array}{c}\infty \\
\stackrel{+}{+} \\
ம^{2}\end{array}$ & $\begin{array}{c}\infty \\
0 \\
0 \\
10\end{array} \mid$ & $\begin{array}{l}\hat{\varphi} \\
\dot{\tau}\end{array}$ & $\begin{array}{l}\hat{N} \\
\dot{f}\end{array}$ & 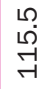 & 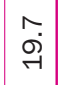 \\
\hline$\nabla$ & ถึ & -1 & \begin{tabular}{l}
$\infty$ \\
\multirow{+}{+}{} \\
$\dot{0}$
\end{tabular} & \begin{tabular}{c}
0 \\
-1 \\
\multirow{1}{1}{} \\
0
\end{tabular} & $\begin{array}{l}8 \\
0 \\
0 \\
0\end{array}$ & $\stackrel{\stackrel{\mathscr{N}}{+}}{0}$ & $\begin{array}{l}\infty \\
\infty \\
ம^{\circ}\end{array}$ & $\begin{array}{l}\infty \\
\stackrel{\infty}{+} \\
\iota^{\circ}\end{array}$ & $\begin{array}{l}\infty \\
0 \\
0 \\
10\end{array} \mid$ & $\begin{array}{l}\hat{\varphi} \\
\dot{\sigma}\end{array}$ & $\begin{array}{l}\hat{N} \\
+\end{array}$ & $\begin{array}{l}0 \\
\infty \\
\infty \\
0 \\
-1\end{array}$ & $\begin{array}{l}\stackrel{n}{-} \\
\stackrel{-}{-}\end{array}$ \\
\hline$m$ & 7 & -1 & $\begin{array}{l}\hat{\gamma} \\
\dot{J} \\
\dot{0}\end{array}$ & $\begin{array}{c}0 \\
-1 \\
\\
0\end{array}$ & $\begin{array}{l}\mathscr{8} \\
\stackrel{0}{0} \\
\dot{0}\end{array}$ & $\begin{array}{l}\stackrel{\infty}{N} \\
\dot{0}\end{array}$ & $\begin{array}{l}\infty \\
\infty \\
\omega^{\circ}\end{array}$ & $\begin{array}{c}\infty \\
\stackrel{+}{+} \\
\stackrel{1}{0}\end{array}$ & $\begin{array}{l}\hat{0} \\
\dot{0}\end{array}$ & $\begin{array}{l}\hat{\varphi} \\
\dot{\tau}\end{array}$ & 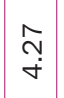 & 守 & $\begin{array}{l}N \\
\dot{\theta}\end{array}$ \\
\hline (2) & & \lrcorner & $\Sigma$ & $\infty$ & $\begin{array}{l}\stackrel{F}{+} \\
\text { の }\end{array}$ & 喵 & 吉 & 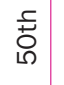 & 㔛 & 故 & के & \multirow[b]{2}{*}{ 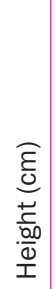 } & \multirow[b]{2}{*}{ 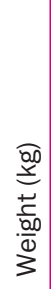 } \\
\hline 遂 & 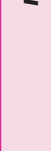 & \multicolumn{10}{|c|}{ 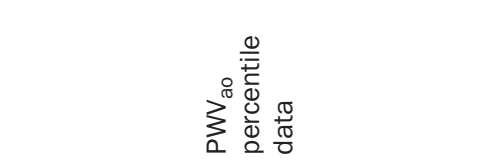 } & & \\
\hline
\end{tabular}

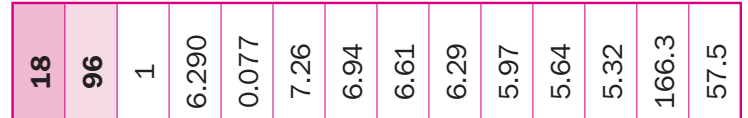

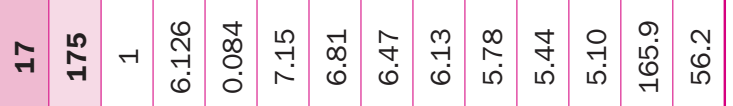

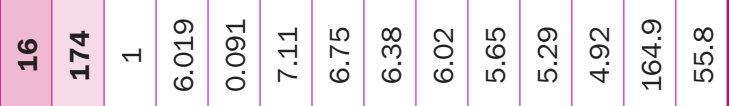

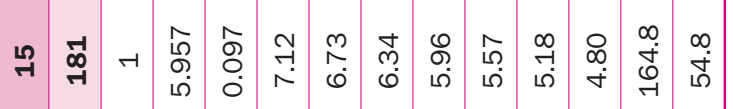

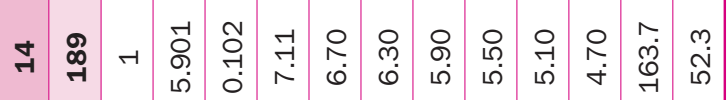

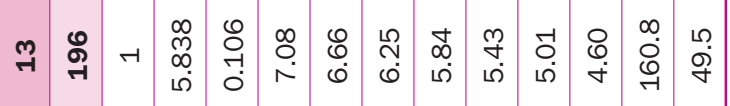
ᄀ

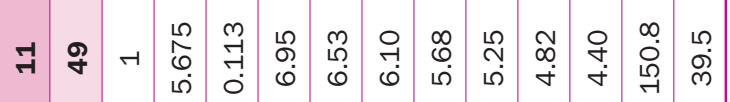

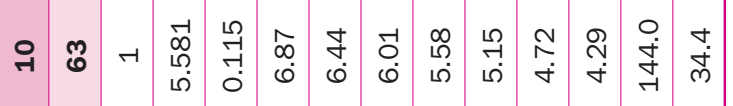

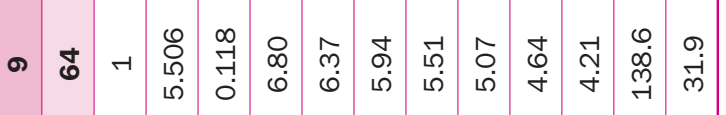

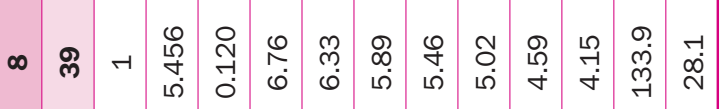

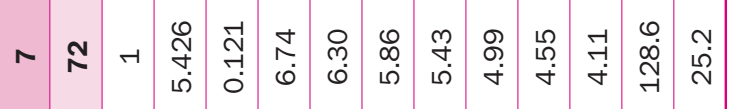

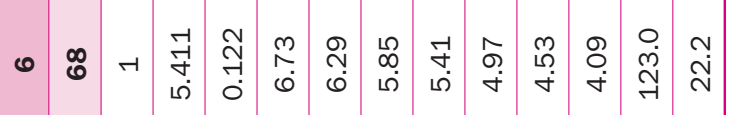

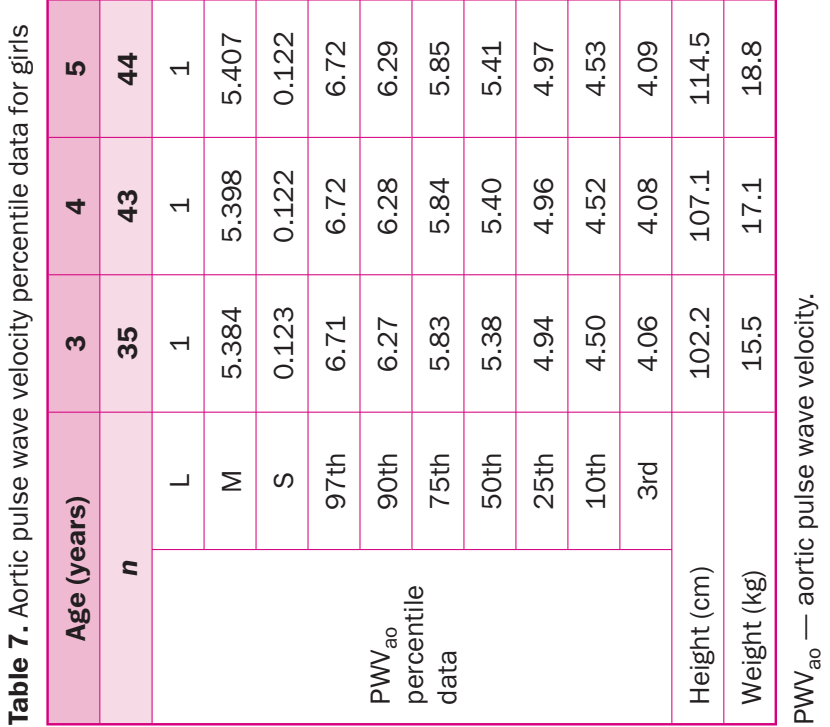


FIGURE 2. Smoothed median curve (pink curve) and first derivative of smoothed median curve of aortic pulse wave velocity $\left(\mathrm{PWV}_{\mathrm{ao}}\right.$; lilac curve) related to age for boys $(\mathrm{A})$ and girls $(\mathrm{B})$

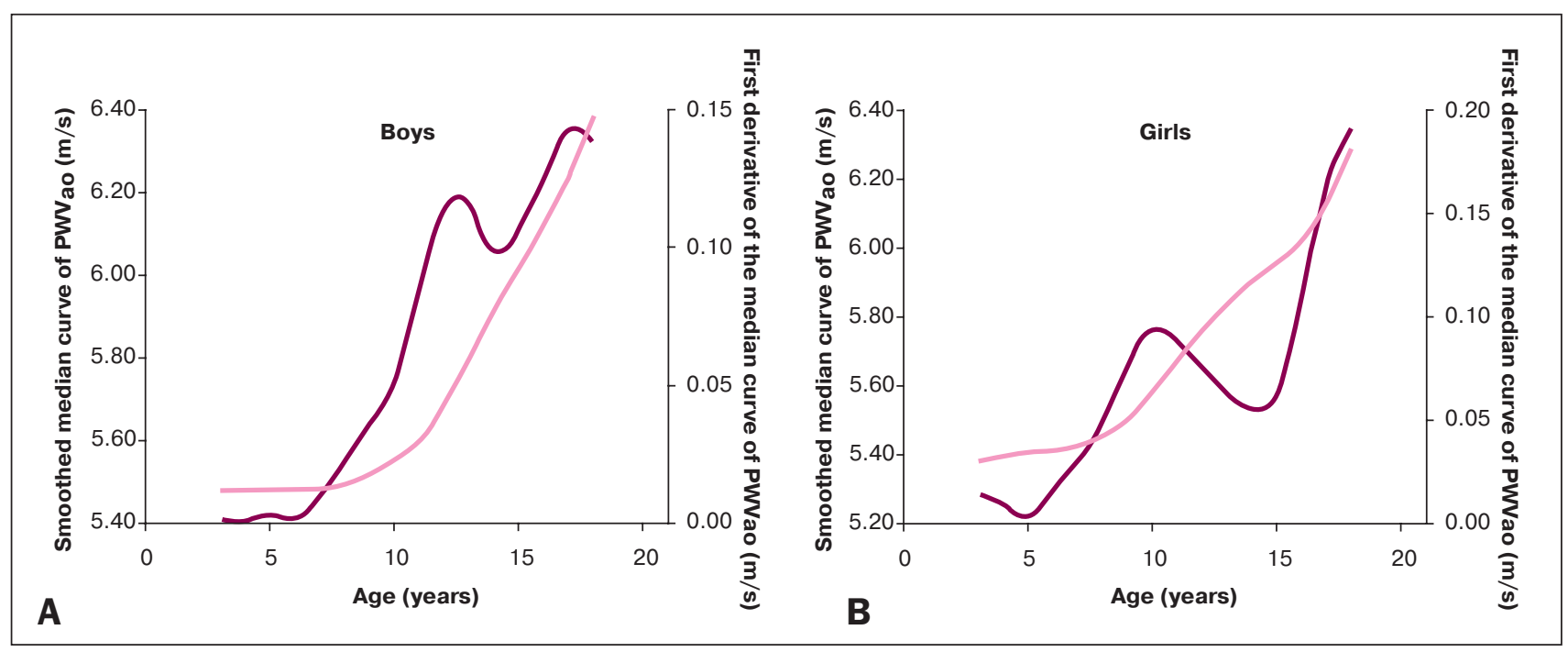

FIGURE 3. Standardized median curves of aortic pulse wave velocity ( $\left.P W V_{a 0}\right)$, mean arterial pressure (MAP) and brachial SBP related to age for boys $(A)$ and girls $(B)$

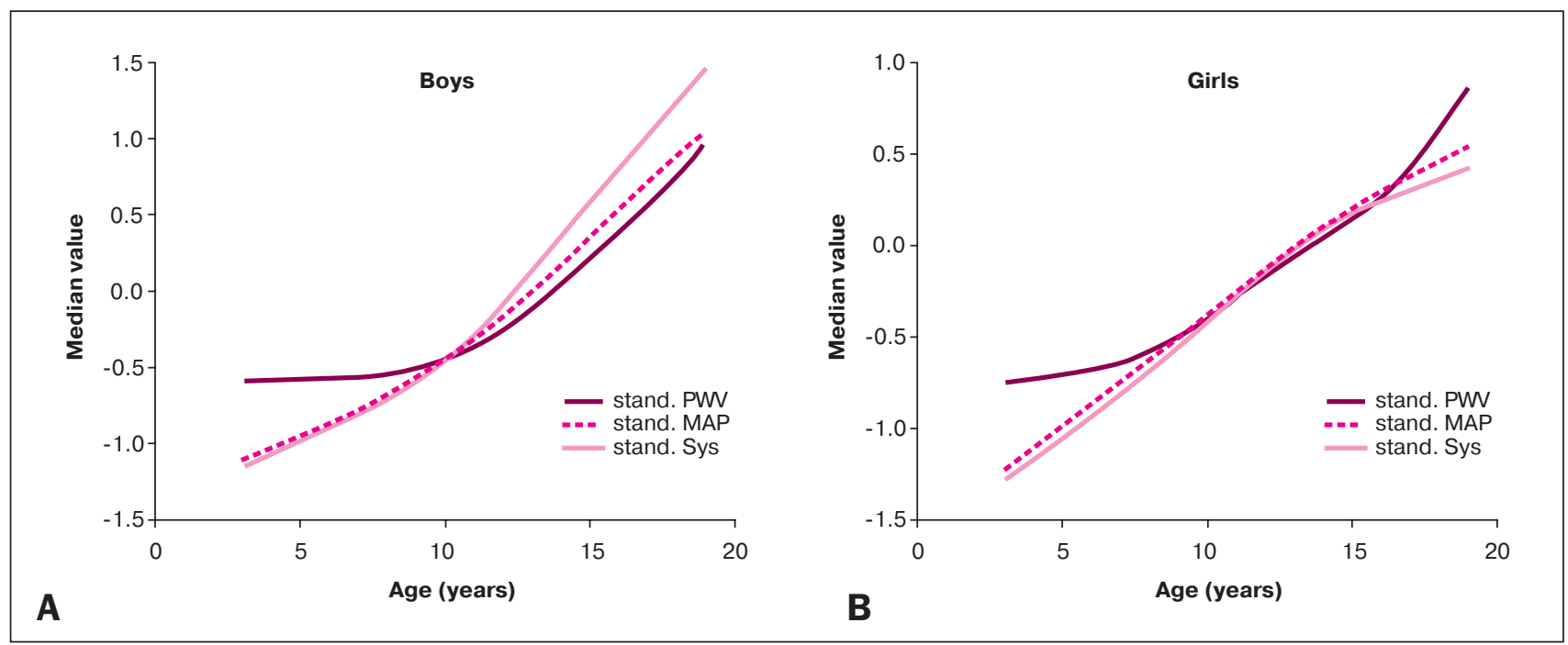

changes in $\mathrm{SBP}_{\text {brach }}$ and MAP versus $\mathrm{PWV}_{\mathrm{ao}}$. Although the blood pressure increased continuously, the $\mathrm{PWV}_{\text {ao }}$ remained practically unchanged in this age range. However, beyond 9 years of age, the blood pressure and aortic stiffness trends basically travelled together.

\section{DISCUSSION}

The most important finding of our study is that we were able to determine the reference values of aortic stiffness on the largest database to date of healthy children and adolescents between the ages of 3 and 18 years. Our homogeneous white population was fairly well balanced in terms of both age and sex.

More importantly, a special characteristic of our database is that it contains a large number of very young subjects ( $<6$ years), as the applied oscillometric, occlusive method (Arteriograph) allowed us to perform the measurements even in this special population. The operational procedure of this device basically does not differ from a standard digital blood pressure measurement and lasts only $2 \mathrm{~min}$. It is a very rapid and painless procedure and is well tolerated even by the youngest participants.

The small size of the portable device enabled us to visit kindergartens and schools to collect the database very easily. Consequently, the adoption of this technology for routine paediatric practice seems to be realistic, especially in view of the user independence of the method.

The measurement of $\mathrm{PWV}_{\mathrm{ao}}$ of the used method is based on the assumption that the reflection site of the first systolic aortic pulse wave is at the area of the aortic bifurcation. Horvath et al. [29] clearly demonstrated that the $\mathrm{PWV}_{\mathrm{ao}}$ measured invasively and oscillometrically were very close $(<0.1 \mathrm{~m} / \mathrm{s})$ to each other, suggesting that the major reflection site must be at the area of the bifurcation. As far as the potential change of the position of the aortic bifurcation during growth is concerned, on the basis of our literature search we could not find any data about the change of the position of the aortic bifurcation related to its vertebral position during growing between ages of 3 and 18 years. In the available anatomical textbooks the 
length and the position of aortic parts (thoracic, abdominal) are determined in the relationship with vertebral number (thoracic or abdominal). Minor changes in the position of the aortic bifurcation, related to its vertebral position during growing cannot be excluded theoretically. Furthermore, this kind of potential bias due to the growing may exist if the measurement of the aortic PWV would be performed by carotid-femoral method.

The overall increase with age of mean $\mathrm{PWV}_{\mathrm{ao}}$ values was identical in both sexes, only biologically insignificant differences were observed between them. These findings suggest that no significant structural difference exists between sexes in this age range.

Regarding the augmentation index, Ayer et al. measured carotid augmentation index (Alx) in 8-year-old children to examine the influences of sex, height and arterial stiffness on central arterial pulse wave augmentation [34]. They found that carotid Alx was significantly higher in girls than boys and it was independent of height, carotid artery diameter and stiffness. In contrast in our study we focused on the PWV and proved that the $\mathrm{PWV}_{\text {ao }}$ does not increase constantly with age. In the very young age groups (between 3 and 8 years), the median $\mathrm{PWV}_{\mathrm{ao}}$ values are practically constant for both sexes.

The second original finding was that we observed a very important (previously not described) characteristic of the smoothed percentile curves of $\mathrm{PWV}_{\mathrm{ao}}$ values.

During a detailed and careful analysis when the first derivatives of the smoothed median curve of $\mathrm{PWV}_{\mathrm{ao}}$ were applied, it emerged that the steepest increase differs in terms of time between boys and girls, that is it occurs at 12.1 years in boys and at 10.4 years in girls. We can hypothesize that this newly observed phenomenon might be explained by the differing onset of adolescence in boys and girls.

When we compared the age-related changes of the median of the standardized values of $\mathrm{PWV}_{\mathrm{a}}$, MAP and $\mathrm{SBP}_{\text {brach }}$, a further new and interesting feature appeared. We observed marked differences between the ages of 3 and 8 years in the trends of $\mathrm{PWV}_{\mathrm{ao}}$, MAP and $\mathrm{SBP}_{\text {brach }}$ Interestingly, the $\mathrm{PWV}_{\mathrm{ao}}$ remained practically unchanged, whereas the blood pressure trend constantly increased. This raises several physiological questions. We might suppose that, at a very young age, the aortic wall is so elastic that an increase in blood pressure does not cause any consequential stiffening in the aortic wall, but, clearly, further, specifically designed studies would be needed to confirm this hypothesis. Similarly the practically identical increase of the standardized $\mathrm{PWV}_{\mathrm{a}}$ and peripheral blood pressure at the later ages of 10-18 years might be due to a gradual loss of aortic elastic properties.

Several authors have provided evidence about the changes in the structure and composition of the aortic wall at an early age, specifically that ageing causes a loss in elastic properties [35-39]. It is possible that the continuously increasing blood pressure which occurs between the ages of 3 and 18 years may lead to a simultaneous increase in lateral tension in the aortic wall. Then beyond the age of 10-12 years, this load cannot be compensated for as successfully as was the case between 3 and 8 years. Hence, the rise in pressure will inevitably be accompanied by a rise in aortic stiffness.

Whether the abnormally steep increase of $\mathrm{PWV}_{\mathrm{ao}}$ (e.g. an individual's increase in $\mathrm{PWV}_{\mathrm{ao}}$ from the 25th to 90th percentile within a few years) might have pathological value in assessing cardiovascular risk characterized by the accelerated stiffening of the aortic wall is something which remains to be clarified by further, preferably longitudinal examination.

Assessing our age-related mean $\mathrm{PWV}_{\mathrm{ao}}$ values in the light of earlier studies, the observed absolute values and their changes in relation to age seem to be very similar, suggesting that the $\mathrm{PWV}_{\mathrm{ao}}$ values provided by the oscillometric method fall strictly within the range found by other examiners (Table 1). Unfortunately, a more detailed comparison of the observed mean $\mathrm{PWV}_{\mathrm{ao}}$ values between studies cannot be undertaken, as neither the values of the measured distances nor the transit times of the pulse pressure waves between the observational points are given by the cited authors.

In this respect, our measured mean $\mathrm{PWV}_{\text {ao }}$ values (5.5$6.5 \mathrm{~m} / \mathrm{s}$ in boys and $5.6-6.4 \mathrm{~m} / \mathrm{s}$ in girls) are closest to the results of Reusz et al. published recently, although the upper age of their participants was 20 years [24]. Their applied distance measurement [(femoral site-jugular notch) (carotid site-jugular notch)] and our distance [Jug-Sy, the distance between the sternal notch (jugulum) and upper part of the pubic bone (symphysis)] differs markedly. To be able to judge the difference in distance and to be able to compare the results more precisely, we carried out a small sub-study on our population involving 56 subjects aged between 6 and 18 (quite well distributed in terms of age). We measured the distances in the same subject by both methods and found a difference of $4.6 \mathrm{~cm}$, i.e. the Jug-Sy distance was $4.6 \mathrm{~cm}$ longer on average than the [(femoral site-jugular notch) (carotid site-jugular notch)] distance. Consequently, the $\mathrm{PWV}_{\mathrm{ao}}$ differences between the data of Reusz et al. [24] and of ourselves (roughly $1 \mathrm{~m} / \mathrm{s}$ ) originated basically from the different methods which were used.

To summarize our study, we provided $\mathrm{PWV}_{\mathrm{ao}}$ reference values for 3374 healthy whites, reasonably balanced in terms of age from 3 to 18 years, using a valid and reliable technique which can be used even at very young ages. We first detailed the specific characteristics of the changes of $\mathrm{PWV}_{\mathrm{ao}}$ - namely that the $\mathrm{PWV}_{\mathrm{ao}}$ remains unchanged in the very young (despite increasing blood pressure), and we found the gradual increase of $P W V_{a o}$ in adolescence - which is proportionate to the increase in blood pressure.

\section{Study limitations}

Our data are collected only in white population with no any ethnic or race mixture. Data collection in other population may be the issue of future researches.

\section{ACKNOWLEDGEMENTS}

The authors thank Janos Hubert, Gyorgyi Jakli, Ilona Kelemen, Hajnalka Klemencz (data collection) and Gabor ERSEK (data analysis) for their contribution to this work.

This study was supported by the Hungarian National Research Foundation (OTKA) No.78480.

\section{Conflicts of interest}

M.I. owns shares in TensioMed Ltd., the company which manufactures the Arteriograph and other devices for measuring vascular stiffness. F.T.M. is employed at TensioMed Ltd. The other authors have declared no conflicts of interest. 


\section{REFERENCES}

1. Mancia G., De Backer G., Dominiczak A. et al. 2007 ESH-ESC practice guidelines for the management of arterial hypertension: ESH-ESC Task Force on the Management of Arterial Hypertension. J. Hypertens. 2007; 25: 1751-1762.

2. Celermajer D.S., Sorensen K.E., Gooch V. M. et al. Noninvasive detection of endothelial dysfunction in children and adults at risk of atherosclerosis. Lancet. 1992; 340: 1111- 1115.

3. Meyer A.A., Kundt G., Steiner M. et al. Impaired flow-mediated vasodilation, carotid artery intima-media thickening, and elevated endothelial plasma markers in obese children: the impact of cardiovascular risk factors. Pediatrics. 2006; 117: 1560-1567.

4. McEniery C.M., Bolton C.E., Fawke J. et al. Cardiovascular consequences of extreme prematurity: the EPICure study. J. Hypertens. 2011; 29: 1367-1373.

5. Wiegman A., de Groot E., Hutten B.A., Rodenburg J., Gort J., Bakker H.D. et al. Arterial intima-media thickness in children heterozygous for familial hypercholesterolaemia. Lancet. 2004; 363: 369-370.

6. Riggio S., Mandraffino G., Sardo M. A. et al. Pulse wave velocity and augmentation index, but not intimamedia thickness, are early indicators of vascular damage in hypercholesterolemic children. Eur. J. Clin. Invest. 2010; 40: 250-257.

7. Singh T. P., Groehn H., Kazmers A. Vascular function and carotid intimalmedial thickness in children with insulin-dependent diabetes mellitus. J. Am. Coll. Cardiol. 2003; 41: 661-665.

8. Jarvisalo M.J., Raitakari M., Toikka J. O., Putto-Laurila A., Rontu R., Laine S. et al. Endothelial dysfunction and increased arterial intima-media thickness in children with type 1 diabetes. Circulation. 2004; 109: 1750-1755.

9. Litwin M., Trelewicz J., Wawer Z., Antoniewicz J,. Wierzbicka A., Rajszys P. et al. Intima-media thickness and arterial elasticity in hypertensive children: controlled study. Pediatr. Nephrol. 2004; 19: $767-774$

10. de Divitiis M., Pilla C., Kattenhorn M., Donald A., Zadinello M., Wallace S. et al. Ambulatory blood pressure, left ventricular mass, and conduit artery function late after successful repair of coarctation of the aorta. J. Am. Coll. Cardiol. 2003; 41: 2259-2265.

11. Ou P., Celermajer D.S., Raisky O., Jolivet O., Buyens F., Herment A. et al. Angular (Gothic) aortic arch leads to enhanced systolic wave reflection, central aortic stiffness, and increased left ventricular mass late after aortic coarctation repair: evaluation with magnetic resonance flow mapping. J. Thorac. Cardiovasc. Surg. 2008; 135: 62-68.

12. Sheth R. D., Perez M. D., Goldstein S. L. Cardiovascular calcifications in paediatric patients receiving maintenance dialysis. Pediatr. Nephrol. 2003; 18: 810-813.

13. Charakida M., Donald A.E., Green H., Storry C., Clapson M., Caslake M. et al. Early structural and functional changes of the vasculature in HIVinfected children: impact of disease and antiretroviral therapy. Circulation. 2005; 112: 103-109.

14. Noto N., Okada T., Yamasuge M., Taniguchi K., Karasawa K., Ayusawa M. et al. Noninvasive assessment of the early progression of atherosclerosis in adolescents with Kawasaki disease and coronary artery lesions. Pediatrics. 2001; 107: 1095-1099.

15. Tedesco M.A., Di Salvo G., Ratti G., Natale F., Calabrese E., Grassia C. et al. Arterial distensibility and ambulatory blood pressure monitoring in young patients with neuro-fibromatosis type 1. Am. J. Hyperten. 2001; 14: 559-566.

16. Cheung Y. F., Brogan P.A., Pilla C.B., Dillon M. J., Redington A. N. Arterial distensibility in children and teenagers: normal evolution and the effect of childhood vasculitis. Arch. Dis. Child. 2002; 87: $348-351$

17. Tauzin L., Rossi P., Giusano B., Gaudart J., Boussuges A., Fraisse A. et al. Characteristics of arterial stiffness in very low birth weight premature infants. Pediatr. Res. 2006; 60: 592-596.

18. Kwok K.L., Ng D.K., Cheung Y.F. BP and arterial distensibility in children with primary snoring. Chest. 2003; 123: 1561-1566.

19. Tedesco M. A., Ratti G., Di Salvo G., Martiniello A. R., Limongelli G. Grieco M. et al. Noninvasive evaluation of arterial abnormalities in young patients with neuro-fibromatosis type 1. Angiology. 2000; 51: $733-741$.

20. Ahimastos A.A., Formosa M., Dart A. M., Kingwell B.A. Gender differences in large artery stiffness pre and postpuberty. J. Clin. Endocrinol. Metab. 2003; 88: 5375-5380.
21. Avolio A. P., Deng F. Q., Li W. Q., Luo Y. F., Huang Z. D., Xing L. F. et al. Effects of aging on arterial distensibility in populations with high and low prevalence of hypertension: comparison between urban and rural communities in China. Circulation. 1985; 71: 202-210.

22. Avolio A.P., Chen S.G., Wang R.P., Zhang C.L., Li M.F., O'Rourke M. F. Effects of aging on changing arterial compliance and left ventricular load in a northern Chinese urban community. Circulation. 1983; 68: 50-58.

23. Kis E., Cseprekal O., Horvath Z., Katona G., Fekete B. C. Hrapka E. et al. Pulse wave velocity in end-stage renal disease: influence of age and body dimensions. Pediatr. Res. 2008; 63: 95-98.

24. Reusz G.S., Cseprekal O., Temmar M., Kis E., Cherif A.B., Thaleb A. et al. Reference values of pulse wave velocity in healthy children and teenagers. Hypertension. 2010; 56: 217-224.

25. Urbina E., Williams R., Alpert B.S., Collins R.T., Daniels S.R., Hayman L. et al. on behalf of the American Heart Association Atherosclerosis, Hypertension, and Obesity in Youth Committee of the Council on Cardiovascular Disease in the Young Noninvasive Assessment of Subclinical Atherosclerosis in Children and Adolescents. Recommendations for standard assessment for clinical research: a scientific statement from the American Heart Association. Hypertension. 2009; 54: 919-951.

26. Rosario A. S., Kurth B. M., Stolzenberg H., Ellert U., Neuhauser H. Body mass index percentiles for children and adolescents in Germany based on a nationally representative sample (KiGGS 2003-2006). Eur. J. Clin. Nutr. 2010; 64: 341-349.

27. National High Blood Pressure Education Program Working Group on High Blood Pressure in Children and Adolescents. The fourth report on the diagnosis, evaluation, and treatment of high blood pressure in children and adolescents. Pediatrics. 2004; 114 (2 Suppl 4): 555-576.

28. Baulmann J., Schillings U., Rickert S., Uen S., Dusing R., Illyes M. et al. A new oscillometric method for assessment of arterial stiffness: comparison with tonometric and piezo-electronic methods. J. Hypertens. 2008; 26: 523-528.

29. Horvath I. G., Nemeth A., Lenkey Z., Alessandri N., Tufano F., Kis P. et al. Invasive validation of a new oscillometric device (Arteriograph) for measuring augmentation index, central blood pressure and aortic pulse wave velocity. J. Hypertens. 2010; 28: 2068-2075.

30. Sugawara J., Hayashi K., Yokoi T., Tanaka H. Age-associated elongation of the ascending aorta in adults. J. Am. Coll. Cardiol. Cardiovasc. Imag. 2008; 1: 739-748.

31. Mueller W.H., Martorell R. Reliability and accuracy of measurement. In: Lohman T.G., Roche A.F., Martorell R., editors. Anthropometric standardization reference manual. Champaign Illinois: Human Kinetics Books. 1988. pp. 83-86.

32. Pan H., Cole T.J. LMS Chartmaker, a programme to construct growth references using the LMS method. 2010. Version 2.43. http://www.healthforallchildren.co.uk/.

33. Cole T.J., Green P.J. Smoothing reference centile curves: the LMS method and penalised likelihood. Stat. Med. 1992; 11: $1305-1319$.

34. Ayer J.G., Harmer J.A., Marks G. B., Avolio A. Central arterial pulse wave augmentation is greater in girls than boys, independent of height. J. Hypertens. 2010; 28: 306-313.

35. Martyn C. N., Greenwald S.E. Impaired synthesis of elastin in walls of aorta and large conduit arteries during early development as an initiating event in pathogenesis of systemic hypertension. Lancet. 1997; 350: 953-955.

36. Glukhova M.A., Frid M.G., Koteliansky V.E. Phenotypic changes of human aortic smooth muscle cells during development and in the adult vessel. Am J Physiol. 1991; 261 (4 Suppl.): 78-80.

37. Van Meurs-Van Woezik H., Klein H. W., Markus-Silvis L., Krediet P. Comparison of the growth of the tunica media of the ascending aorta, aorta isthmus and descending aorta in infants and children. J. Anat. 1983; 136: 273-281.

38. Senzaki H., Akagi M., Hishi T., Ishizawa A., Yanagisawa M., Masutani S. et al. Age-associated changes in arterial elastic properties in children. Eur. J. Pediatr. 2002; 161: 547-551.

39. Niwa K., Perloff J.K., Bhuta S.M., Laks H., Drinkwater D.C., Child J.S. et al. Structural abnormalities of great arterial walls in congenital heart disease: light and electron microscopic analyses. Circulation. 2001; 103: 393-400. 